Supporting information

\title{
Magnetic field-induced alignment of nanofibrous supramolecular membranes: a molecular design approach to create tissue-like biomaterials
}

Elham Radvar ${ }^{\dagger}$, Yejiao Shi ${ }^{\dagger}$, Salvatore Grasso ${ }^{\ddagger}$, Charlotte J. C. Edwards-Gayle ${ }^{\S}$, Xitong Liu \#, Meagan S. Mauter ${ }^{\partial}$, Valeria Castelletto ${ }^{\S}$, Ian W. Hamley ${ }^{\S}$. Michael J. Reece ${ }^{\dagger}$ and Helena S. Azevedo $^{\dagger *}$

${ }^{+}$School of Engineering and Materials Science \& Institute of Bioengineering, Queen Mary University of London, Mile End Road, London E1 4NS, United Kingdom

*E-mail: h.azevedo@qmul.ac.uk

${ }^{\ddagger}$ Key Laboratory of Advanced technologies of Materials, Ministry of Education, School of Materials Science and Engineering, Southwest Jiaotong University, Chengdu 61 0031, China

${ }^{\S}$ School of Chemistry, Pharmacy and Food Sciences, University of Reading, Whiteknights, Reading RG6 6AD, United Kingdom

\#Civil \& Environmental Engineering, The George Washington University, 3520 Science and Engineering Hall, 800 22nd St NW, Washington, DC 20052, United States

${ }^{\partial}$ Civil and Environmental Engineering, Stanford University, Y2E2, 473 Via Ortega, Room 311, Stanford, CA 94305, United States 
$\mathrm{Ac}-\mathrm{K}_{2} \mathrm{~A}_{3} \mathrm{~F}_{4}-\mathrm{CONH}_{2}$

Chemical Formula: $\mathrm{C}_{59} \mathrm{H}_{80} \mathrm{~N}_{12} \mathrm{O}_{10}$

Exact Mass: $1116.61 \mathrm{gr} / \mathrm{mol}$
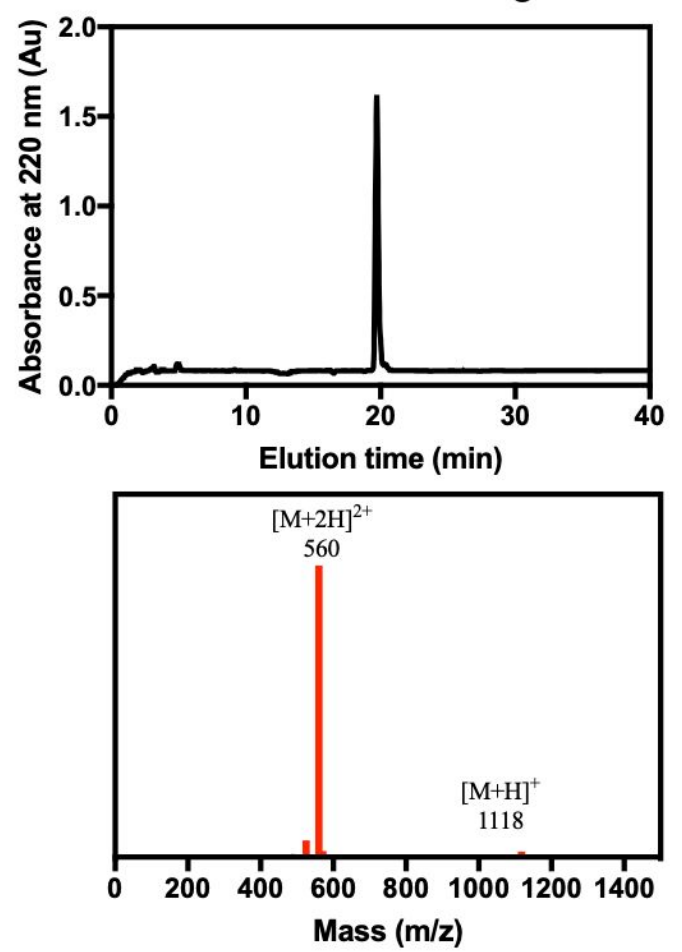

Ac- $\mathrm{K}_{2} \mathrm{~A}_{7} \mathrm{~F}_{4}-\mathrm{CONH}_{2}$

Chemical Formula: $\mathrm{C}_{71} \mathrm{H}_{100} \mathrm{~N}_{16} \mathrm{O}_{14}$

Exact Mass: $1400.76 \mathrm{gr} / \mathrm{mol}$
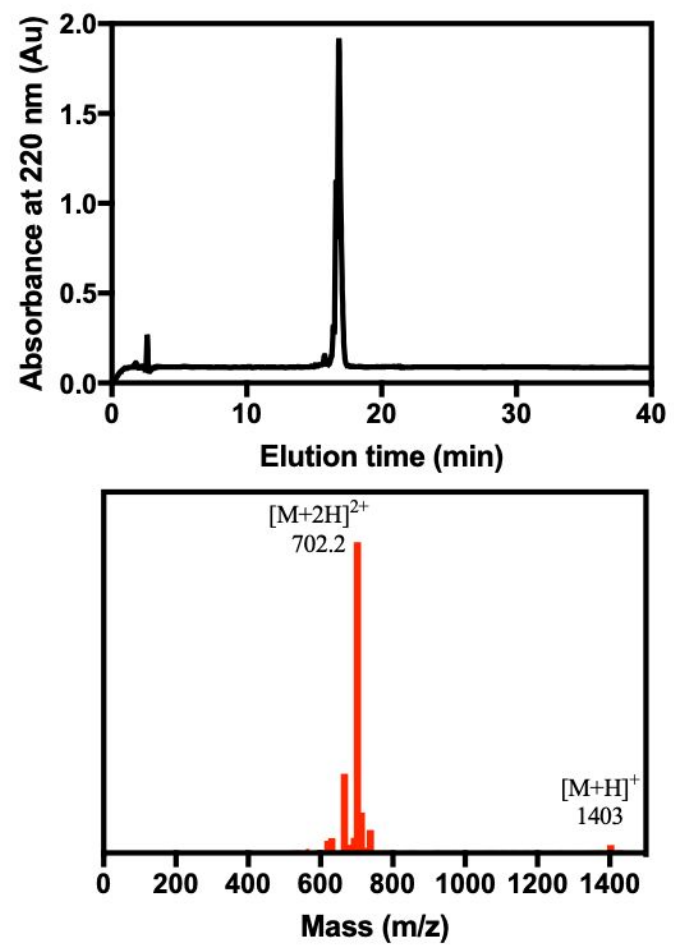

Ac- $\mathrm{K}_{2} \mathrm{~A}_{5} \mathrm{~F}_{4}-\mathrm{CONH}_{2}$

Chemical Formula: $\mathrm{C}_{65} \mathrm{H}_{90} \mathrm{~N}_{14} \mathrm{O}_{12}$

Exact Mass: $1258.69 \mathrm{gr} / \mathrm{mol}$

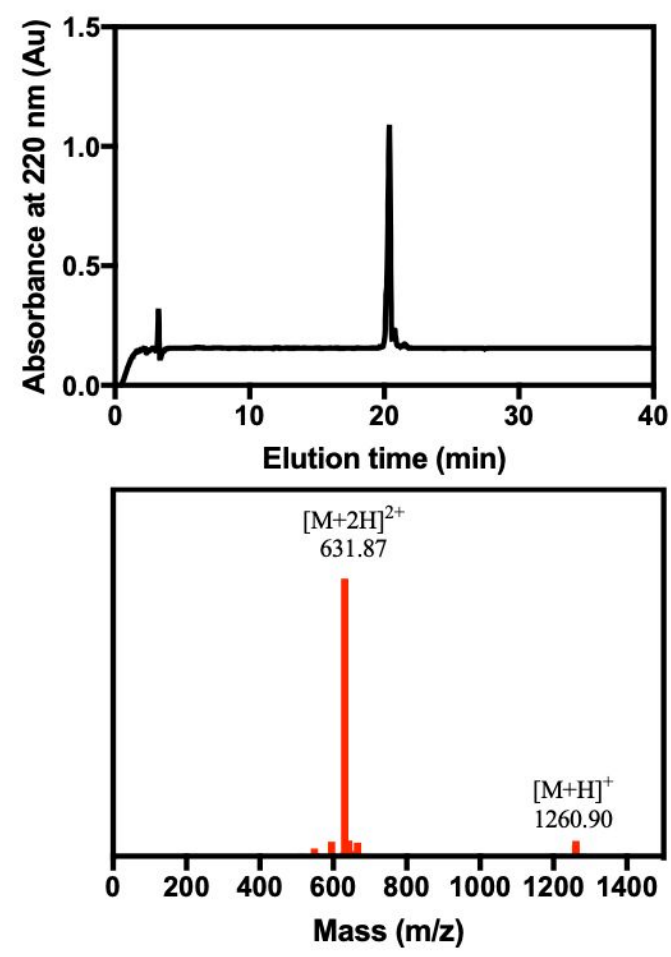

Ac- $\mathrm{K}_{2} \mathrm{~S}_{5} \mathrm{~F}_{4}-\mathrm{CONH}_{2}$

Chemical Formula: $\mathrm{C}_{65} \mathrm{H}_{90} \mathrm{~N}_{14} \mathrm{O}_{17}$ Exact Mass: $1338.66 \mathrm{gr} / \mathrm{mol}$
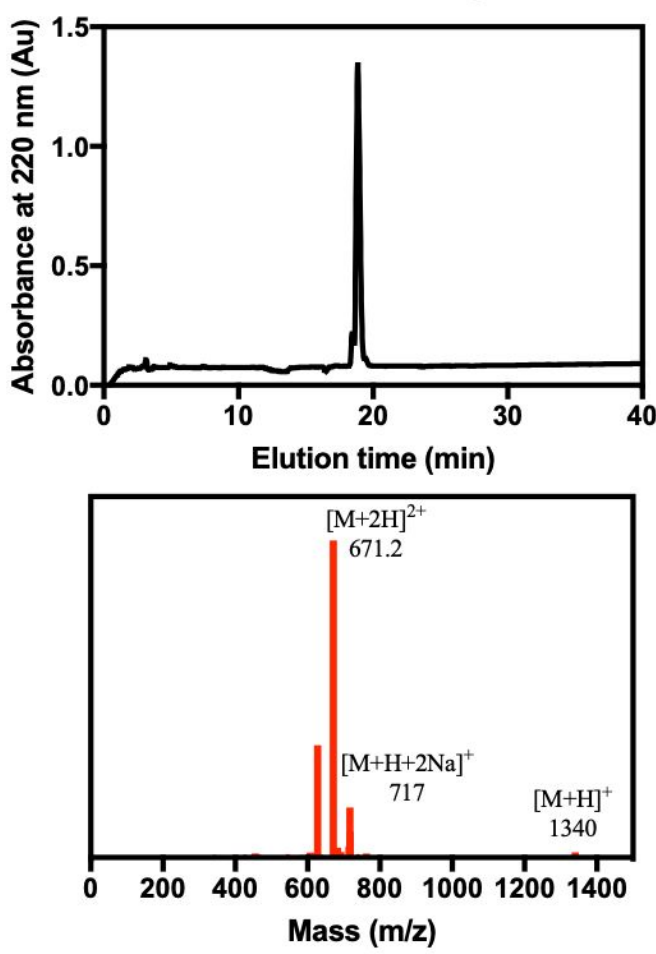
Ac- $\mathrm{K}_{2} \mathrm{G}_{7} \mathrm{~F}_{4}-\mathrm{CONH}_{2}$

Chemical Formula: $\mathrm{C}_{64} \mathrm{H}_{86} \mathrm{~N}_{16} \mathrm{O}_{14}$ Exact Mass: $1302.65 \mathrm{gr} / \mathrm{mol}$
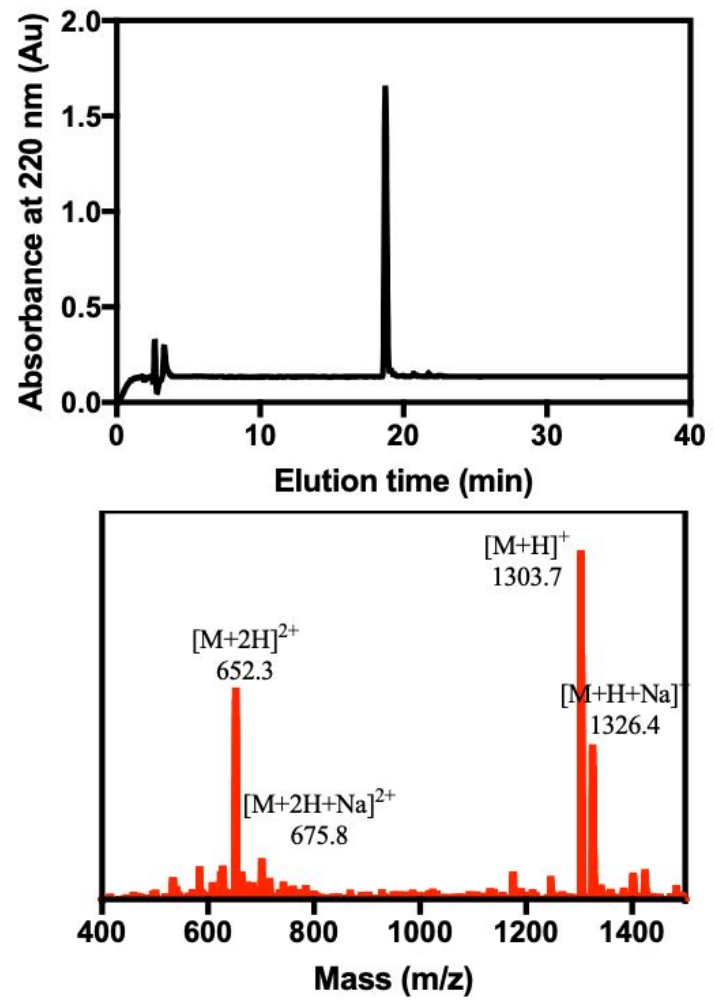

Ac-RGDS- $\mathrm{K}_{2} \mathrm{~A}_{5} \mathrm{~F}_{4}-\mathrm{CONH}_{2}$ Chemical Formula: $\mathrm{C}_{80} \mathrm{H}_{115} \mathrm{~N}_{21} \mathrm{O}_{19}$

Exact Mass: $1673.87 \mathrm{gr} / \mathrm{mol}$
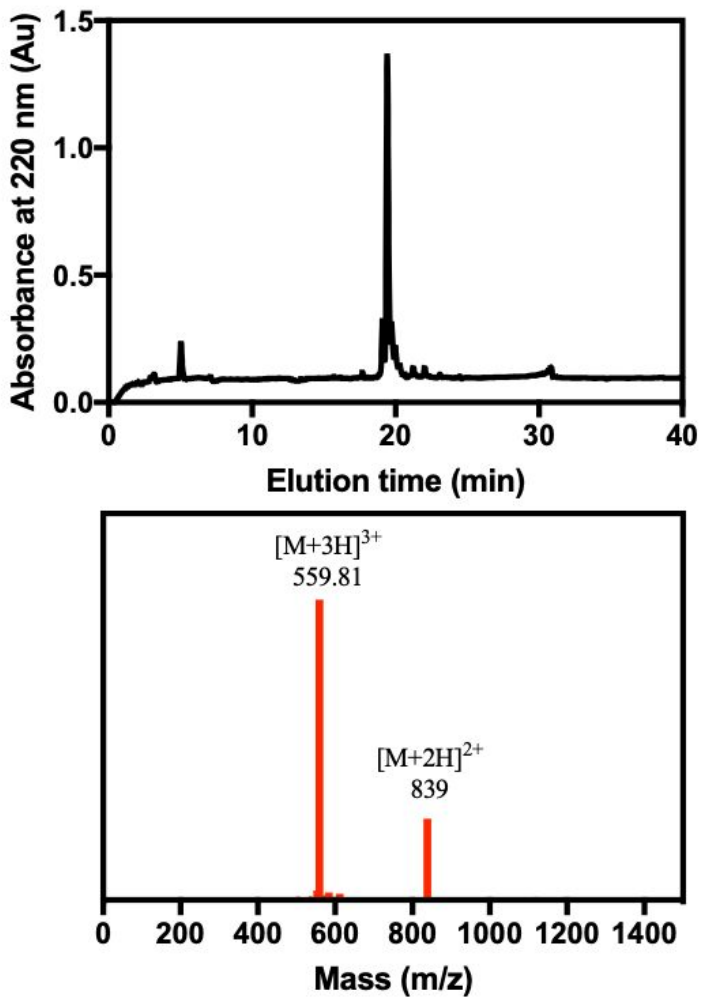

Figure S1. Characterization of cationic aromatic peptides after purification. RP-HPLC traces (top) and ESI-MS spectra (bottom) for each peptide confirming their purity and expected mass.
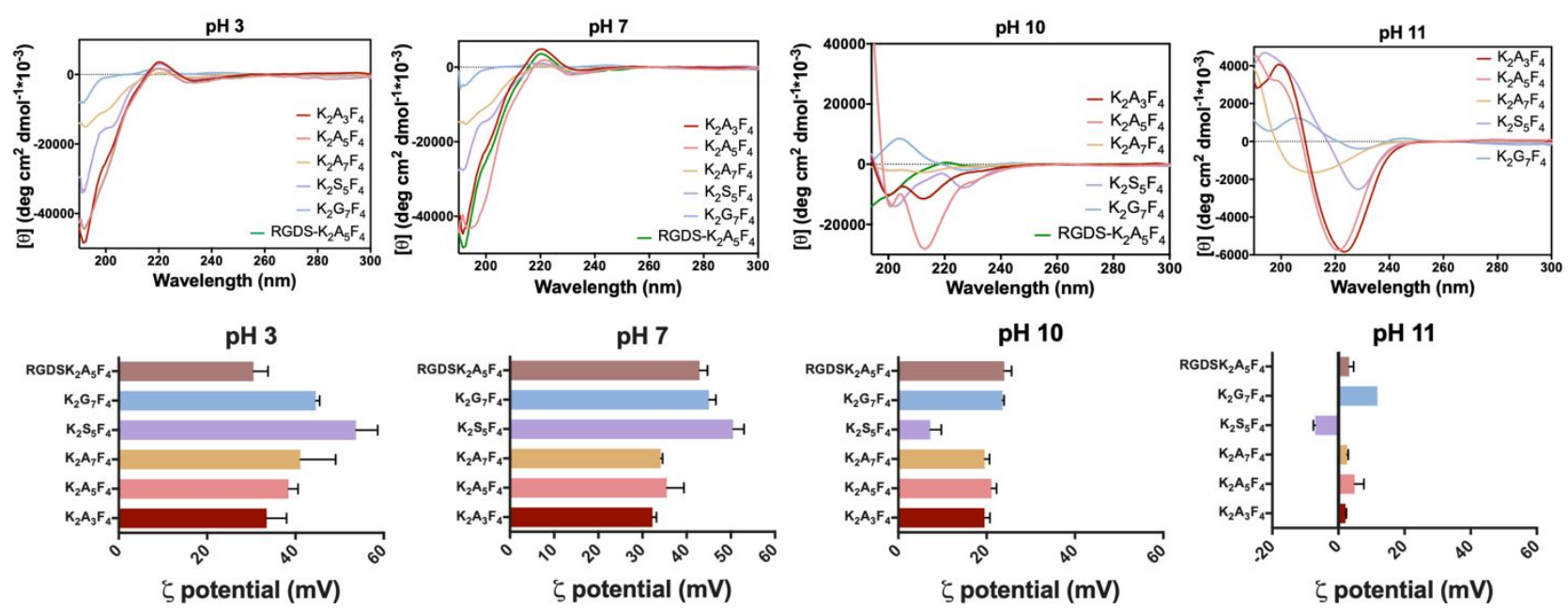

Figure S2. Circular dichroism spectra (top panel) and zeta potential (bottom panel) for all peptides at different $\mathrm{pHs}$. 
Table S1. Calculated diamagnetic anisotropy (DA) of peptides containing different amino acid sequences.

\begin{tabular}{llll} 
& $\begin{array}{c}\text { Molar DA } \\
\left(\times 10^{-3}\right) \mathrm{cm}^{3} / \mathrm{mol}\end{array}$ & \multicolumn{2}{c}{ Volumetric DA $\left(\times 10^{-6}\right)$} \\
$\mathrm{K}_{2} \mathrm{G}_{7} \mathrm{~F}_{5}$ & 0.31 & $\mathrm{~K}_{2} \mathrm{G}_{7} \mathrm{~F}_{5}$ & 4.51 \\
$\mathrm{~K}_{2} \mathrm{G}_{7} \mathrm{~F}_{4}$ & 0.26 & $\mathrm{~K}_{2} \mathrm{G}_{7} \mathrm{~F}_{4}$ & 4.13 \\
$\mathrm{~K}_{2} \mathrm{~S}_{5} \mathrm{~F}_{4}$ & 0.17 & $\mathrm{~K}_{2} \mathrm{~S}_{5} \mathrm{~F}_{4}$ & 3.23 \\
$\mathrm{~K}_{2} \mathrm{~A}_{7} \mathrm{~F}_{4}$ & 0.12 & $\mathrm{~K}_{2} \mathrm{~A}_{7} \mathrm{~F}_{4}$ & 1.98 \\
$\mathrm{~K}_{2} \mathrm{~A}_{5} \mathrm{~F}_{4}$ & 0.07 & $\mathrm{~K}_{2} \mathrm{~A}_{5} \mathrm{~F}_{4}$ & 0.95 \\
$\mathrm{~K}_{3} \mathrm{G}_{5} \mathrm{~F}_{3}$ & 0.04 & $\mathrm{~K}_{3} \mathrm{G}_{5} \mathrm{~F}_{3}$ & 0.78
\end{tabular}

Table S2. Solution SAXS form factor fitting parameters. SAXS fitting was performed using the software SASfit using the Porod cylinder model. ${ }^{[1]}$

\begin{tabular}{|c|c|c|c|c|c|}
\hline Parameter & $\mathbf{K}_{2} \mathbf{A}_{3} \mathbf{F}_{4}$ & $\mathrm{~K}_{2} \mathbf{A}_{5} \mathrm{~F}_{4}$ & $\mathrm{~K}_{2} \mathrm{~A}_{7} \mathrm{~F}_{4}$ & $\mathrm{~K}_{2} \mathrm{~S}_{5} \mathrm{~F}_{4}$ & $\mathbf{K}_{2} \mathrm{G}_{7} \mathbf{F}_{4}$ \\
\hline$R(\AA)^{\mathrm{a}}$ & - & 31.0 & 20.0 & 24.5 & 23.0 \\
\hline$\sigma(\AA)^{b}$ & - & 15.0 & 15.0 & 14.8 & 13.1 \\
\hline$\eta^{c}$ & & $2.5 \times 10^{-7}$ & $3.4 \times 10^{-7}$ & $3.5 \times 10^{-7}$ & $3.0 \times 10^{-7}$ \\
\hline$f^{d}$ & $8.1 \times 10^{-4}$ & $5.0 \times 10^{-4}$ & $4.5 \times 10^{-4}$ & $2.3 \times 10^{-4}$ & $7.2 \times 10^{-5}$ \\
\hline$g^{d}$ & $1.9 \times 10^{-7}$ & $5.3 \times 10^{-7}$ & $9.8 \times 10^{-8}$ & $5.5 \times 10^{-7}$ & $6.0 \times 10^{-7}$ \\
\hline$n^{d}$ & 2.64 & 2.89 & 2.89 & 2.90 & 2.85 \\
\hline
\end{tabular}

${ }^{a}$ Cylinder radius, ${ }^{\mathrm{b}}$ Cylinder radius Gaussian dispersity (HWHM), ${ }^{\mathrm{c}}$ Scattering (relative electron density) contrast, ${ }^{\mathrm{d}}$ Background parameters, background $\mathrm{BG}=f+g q^{- \text {n. }}$. For all fits the cylinder length was fixed to $L=$ $1000 \AA$.

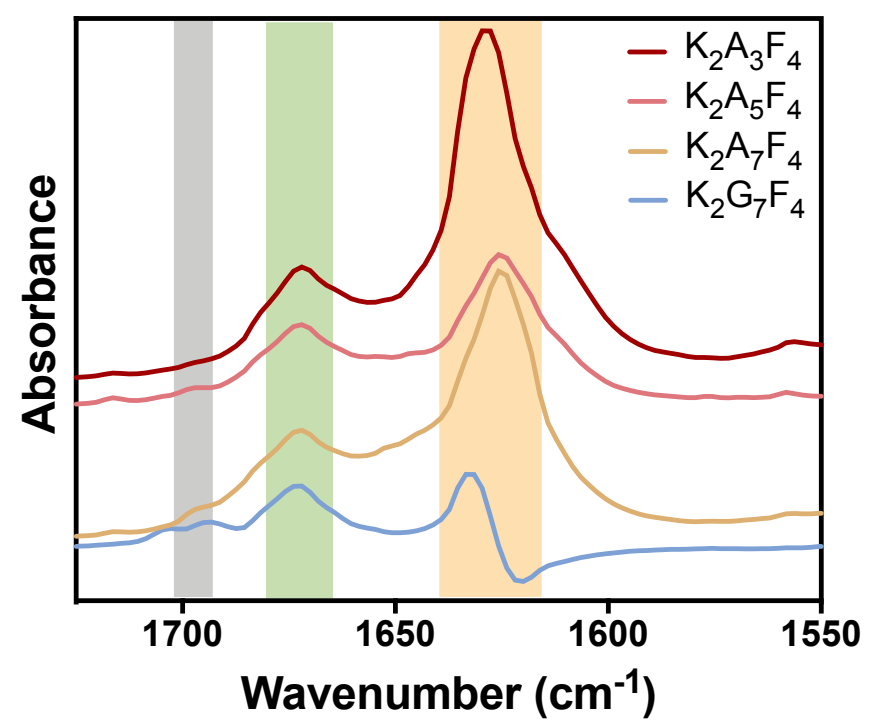

Figure S3. FT-IR spectra of cationic aromatic peptides measured at $\mathrm{pH} 11$ showing parallel $\beta$ sheet (near $1630 \mathrm{~cm}^{-1}$, yellow shade highlight) and antiparallel $\beta$-sheet (peak around $1690 \mathrm{~cm}^{-1}$, grey shade highlight) hydrogen bonding of the peptide backbones. Band at $1670 \mathrm{~cm}^{-1}$ (green shade is attributed to TFA salts) $)^{[2]}$. 
Table S3. FTIR absorbance of the peptides in Amide I region.

\begin{tabular}{|c|c|c|c|}
\hline \multicolumn{4}{|c|}{ FTIR absorbance in the Amide I region $\left(\mathrm{cm}^{-1}\right)$} \\
\hline Peptide & $\boldsymbol{\beta}$-sheet & antiparallel $\boldsymbol{\beta}$-sheet & TFA salt \\
\hline $\mathrm{K}_{2} \mathrm{~A}_{3} \mathrm{~F}_{4}$ & 1627 & - & 1670 \\
\hline $\mathrm{K}_{2} \mathrm{~A}_{5} \mathrm{~F}_{4}$ & 1623 & 1695 & 1670 \\
\hline $\mathrm{K}_{2} \mathrm{~A}_{7} \mathrm{~F}_{4}$ & 1624 & 1697 & 1670 \\
\hline $\mathrm{K}_{2} \mathrm{G}_{7} \mathrm{~F}_{4}$ & 1631 & 1693 & 1670 \\
\hline
\end{tabular}
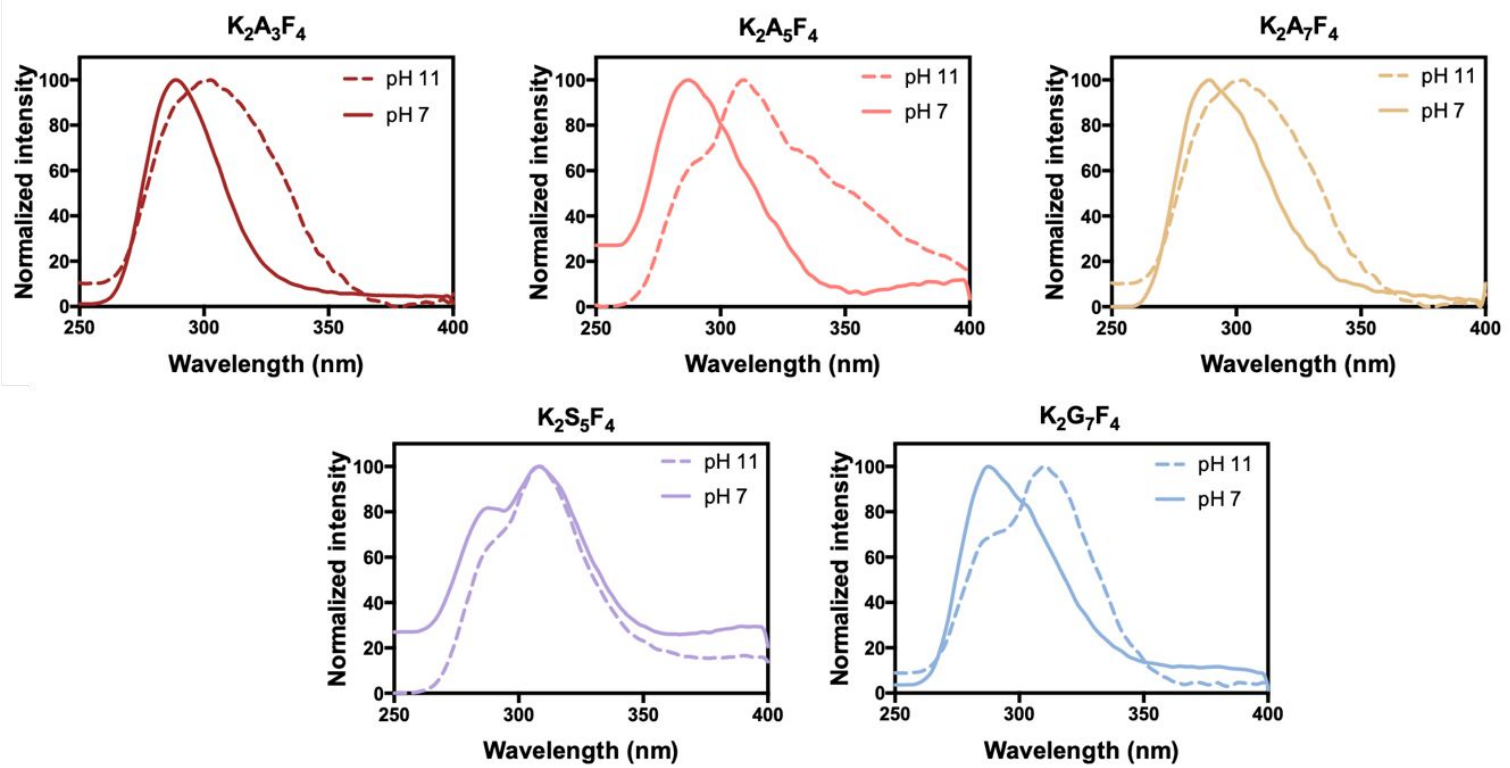

Figure S4. Fluorescence spectra of peptide solutions $(20 \mu \mathrm{M})$ at neutral and basic $\mathrm{pH}$ when excited at $210 \mathrm{~nm}$.
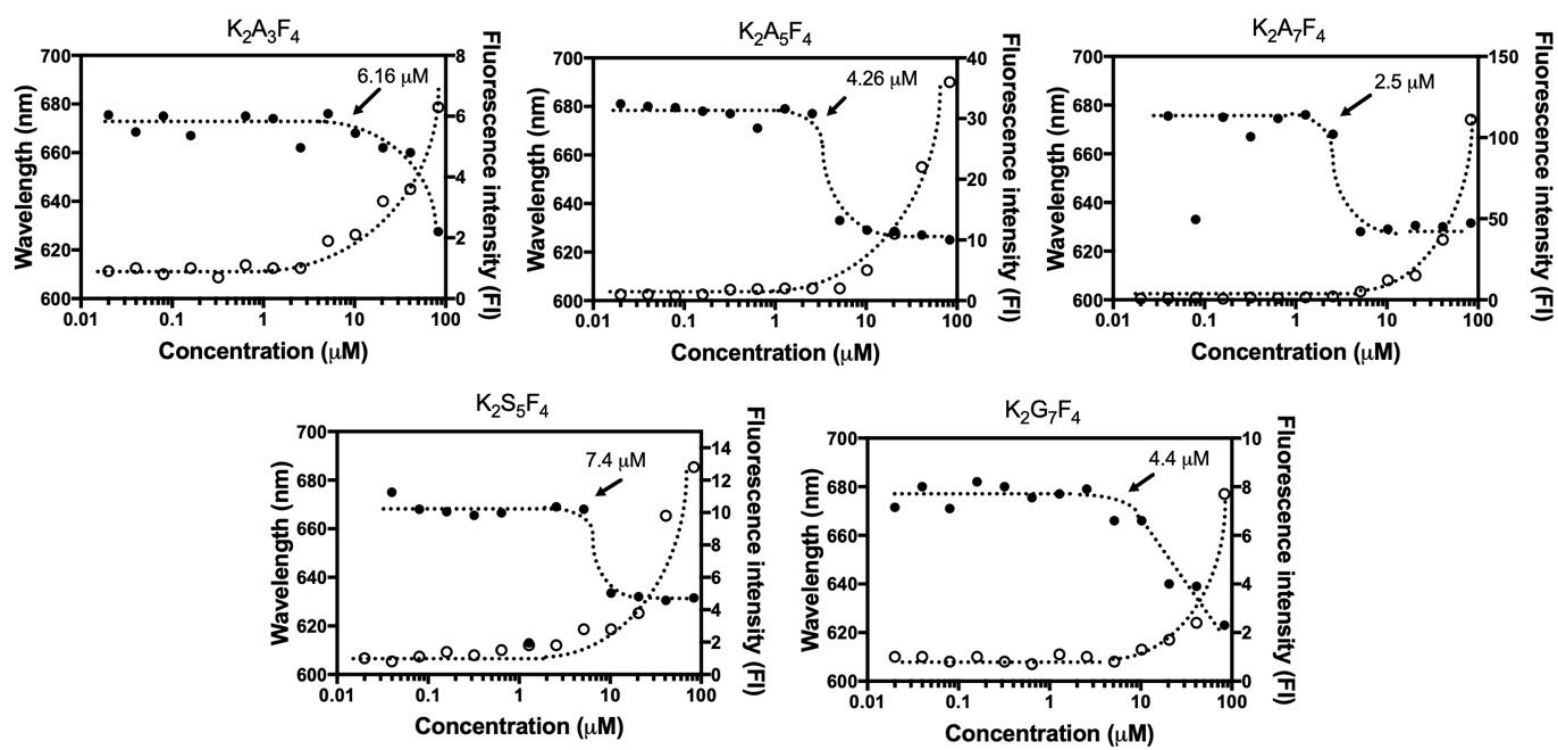

Figure S5. Determination of critical aggregation concentration (CAC) for the different cationic aromatic peptides. Maximum fluorescence emission wavelength and intensity of Nile Red (measured at excitation of Nile Red $(550 \mathrm{~nm})$ and $\mathrm{pH} 7)$ as function of peptide concentration. 
A

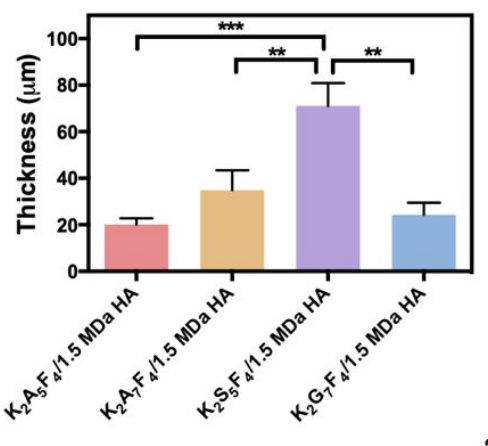

B

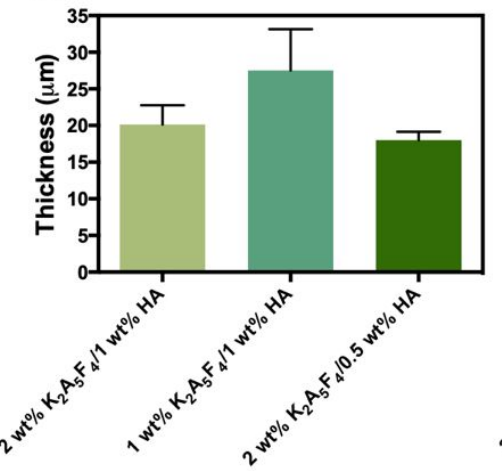

C

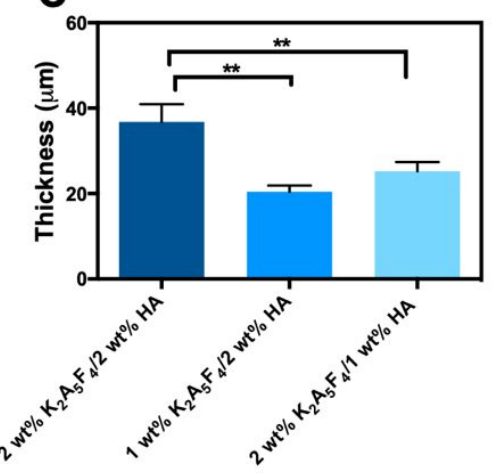

Figure S6. Thickness of self-assembled peptide/HA membranes formed from different cationic aromatic peptides (A) and different concentrations of $1.5 \mathrm{MDa} \mathrm{HA}(\mathrm{B})$ and $700 \mathrm{kDa} \mathrm{HA}(\mathrm{C})$ with $\mathrm{K}_{2} \mathrm{~A}_{5} \mathrm{~F}_{4} .\left({ }^{* *} \mathrm{p}<0.0021,{ }^{* * *} \mathrm{p}<0.0002\right)$.
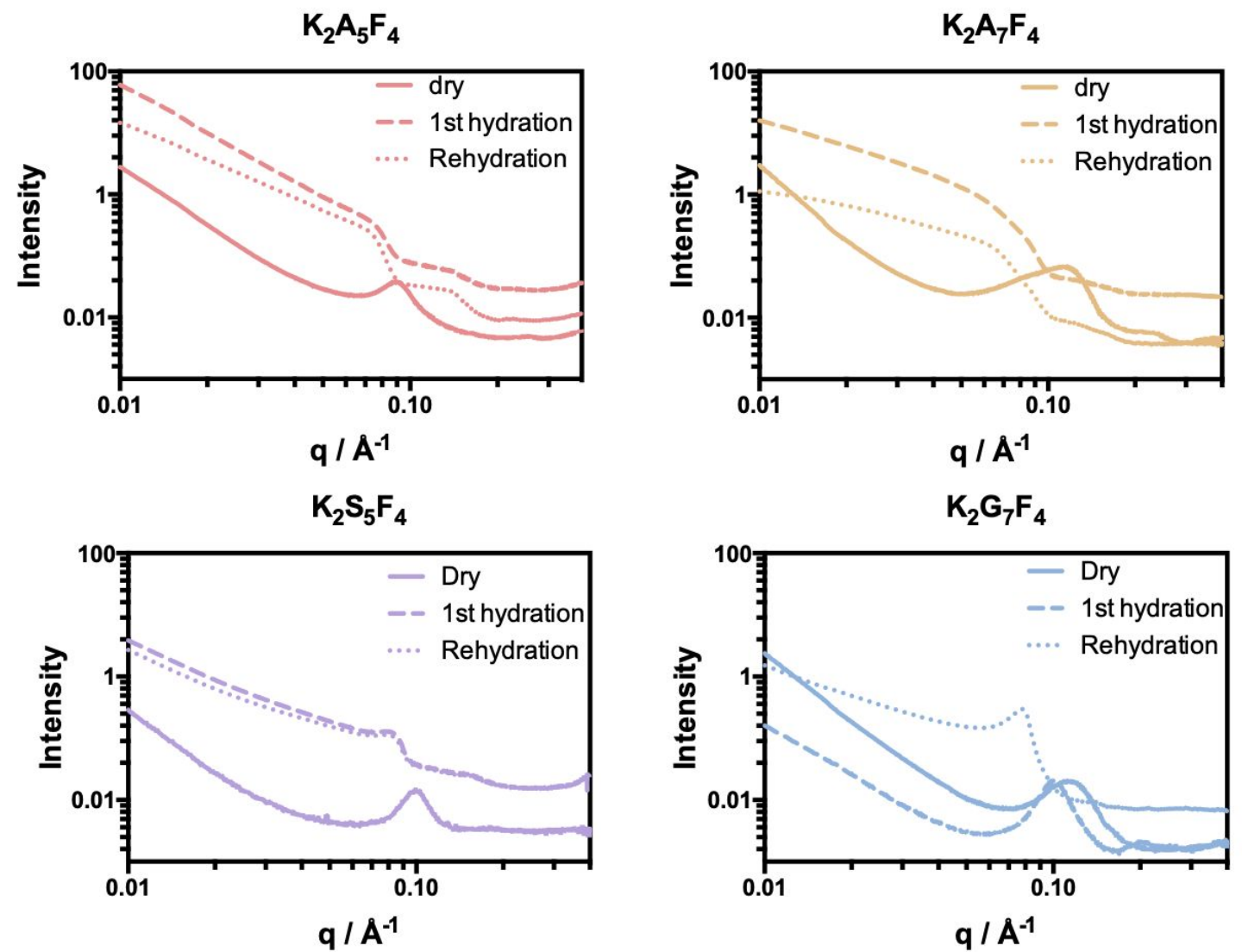

Figure S7. SAXS profiles of self-assembled peptide/HA membranes obtained in the dry (solid line), hydrated (dashed line) and rehydrated (dotted line) states. 


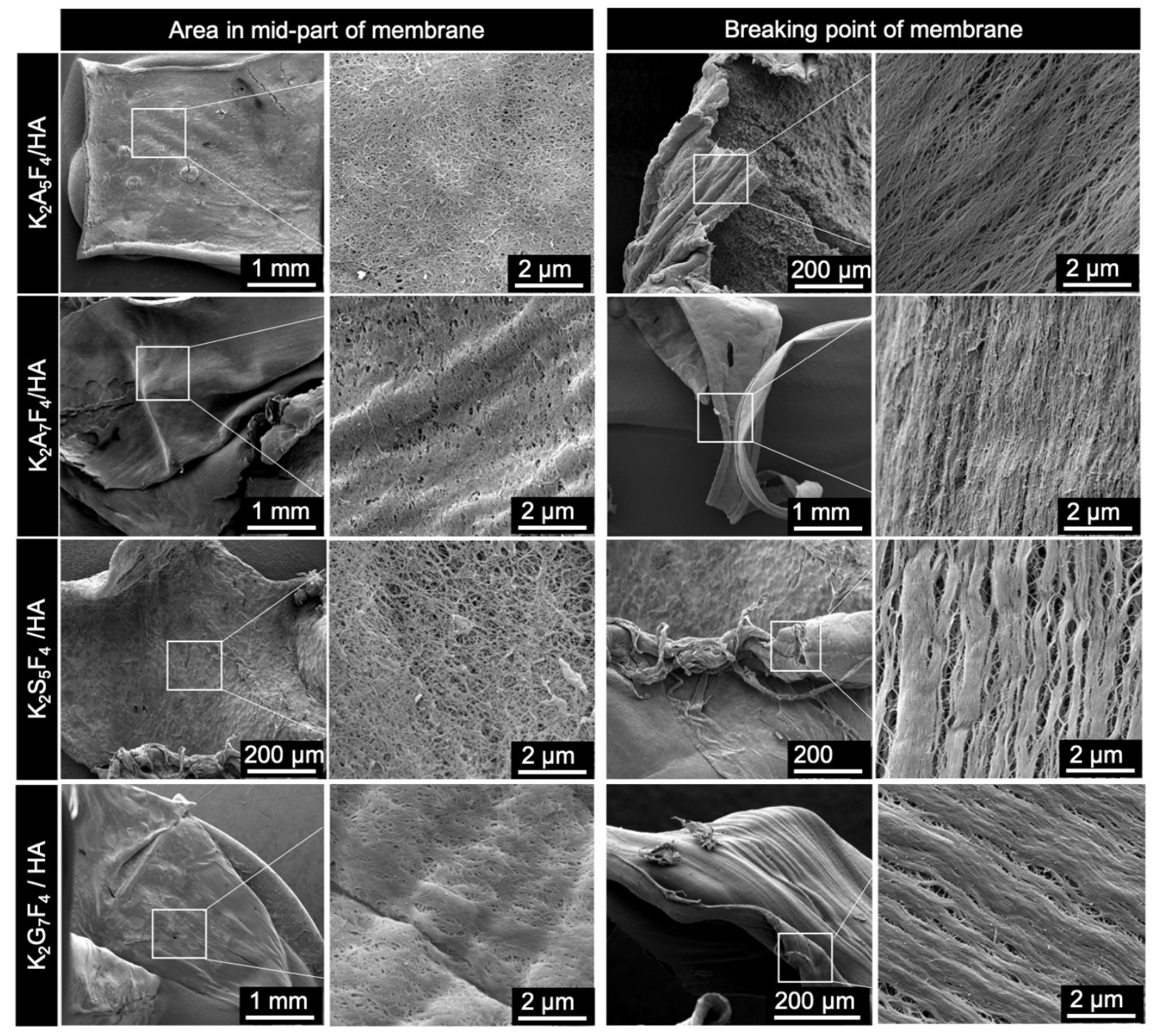

Figure S8. SEM images of membranes after tensile testing showing both areas at the middle and at the breaking point during the test. 
A

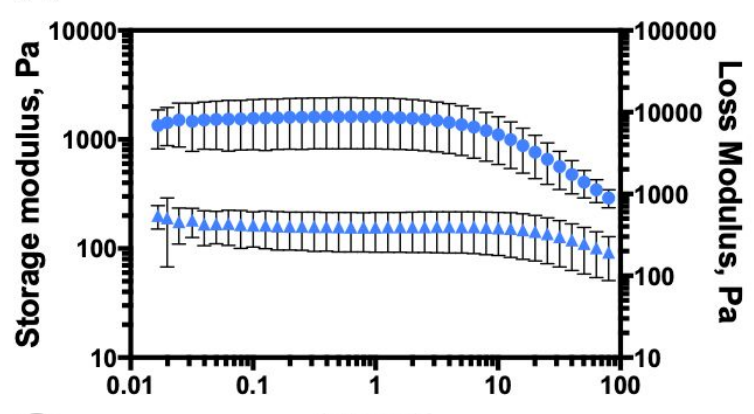

C

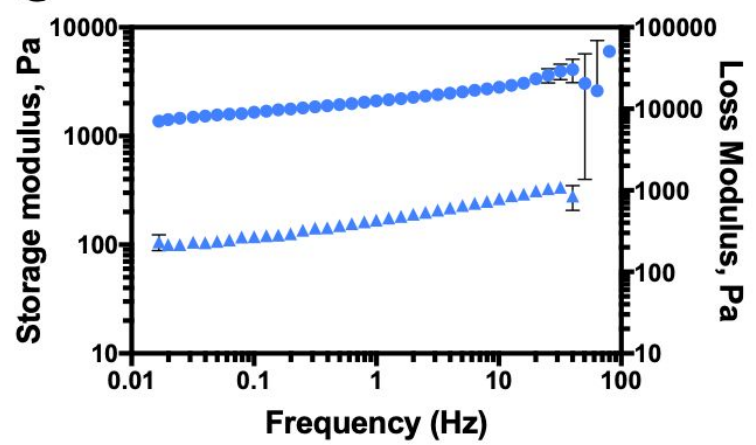

B

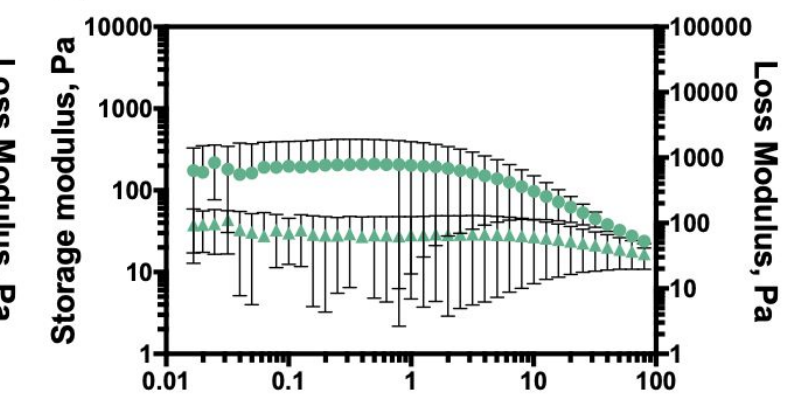

D

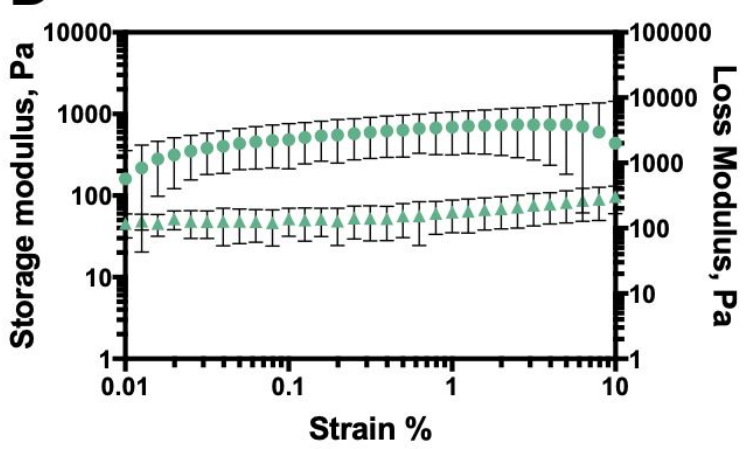

Figure S9. Mechanical properties of $\mathrm{K}_{2} \mathrm{~A}_{3} \mathrm{~F}_{4} / \mathrm{HA}$ hydrogels: (A, B) amplitude sweep and (C, D) Frequency sweep (1.5 MDa HA shown in blue and $700 \mathrm{kDa} H A$ in green).
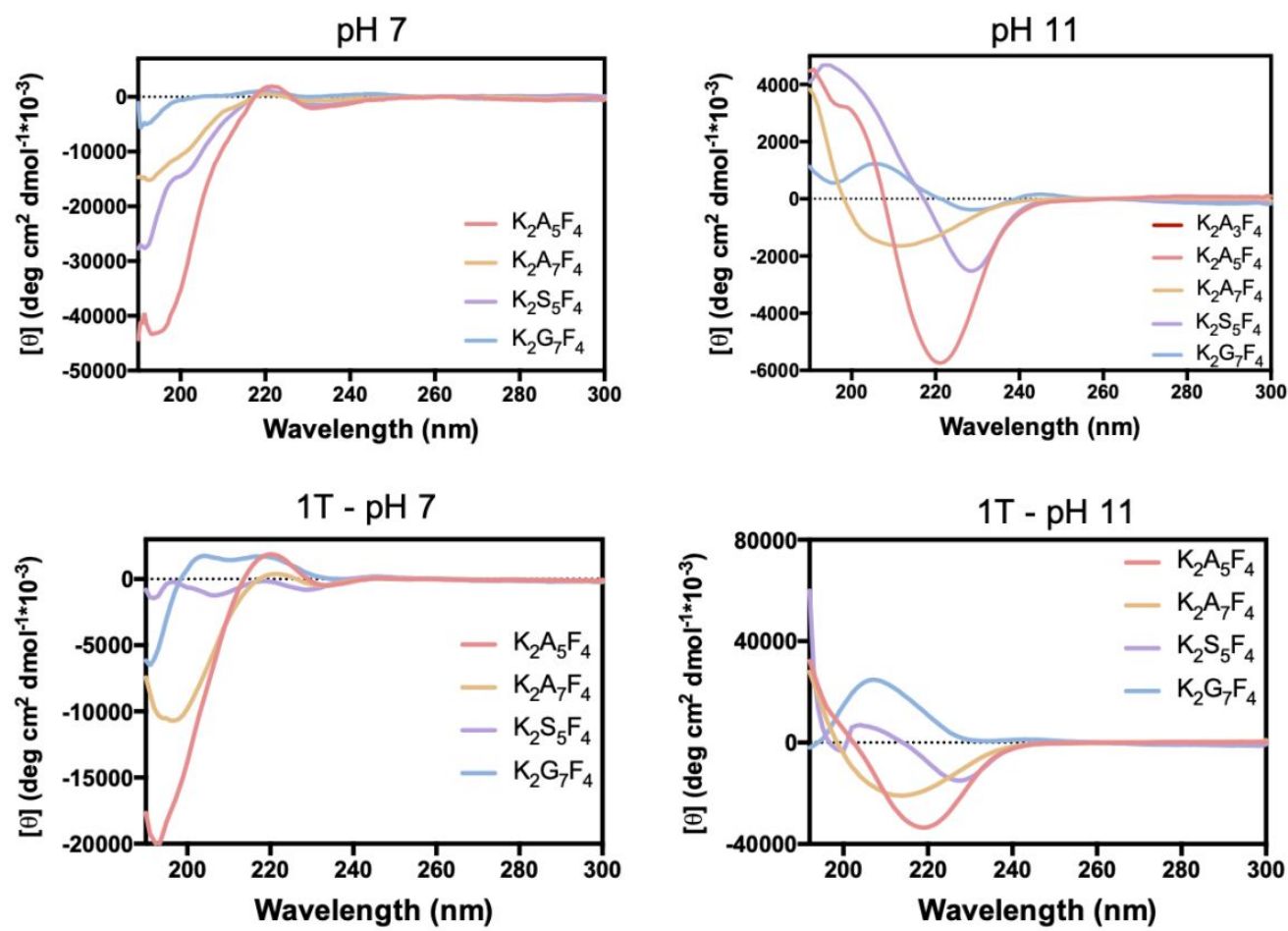

Figure S10. CD spectra comparing the secondary structure of cationic aromatic peptides with and without magnetic field exposure (bottom graphs are obtained after 24 hours incubation under $1 \mathrm{~T}$ magnetic field). 

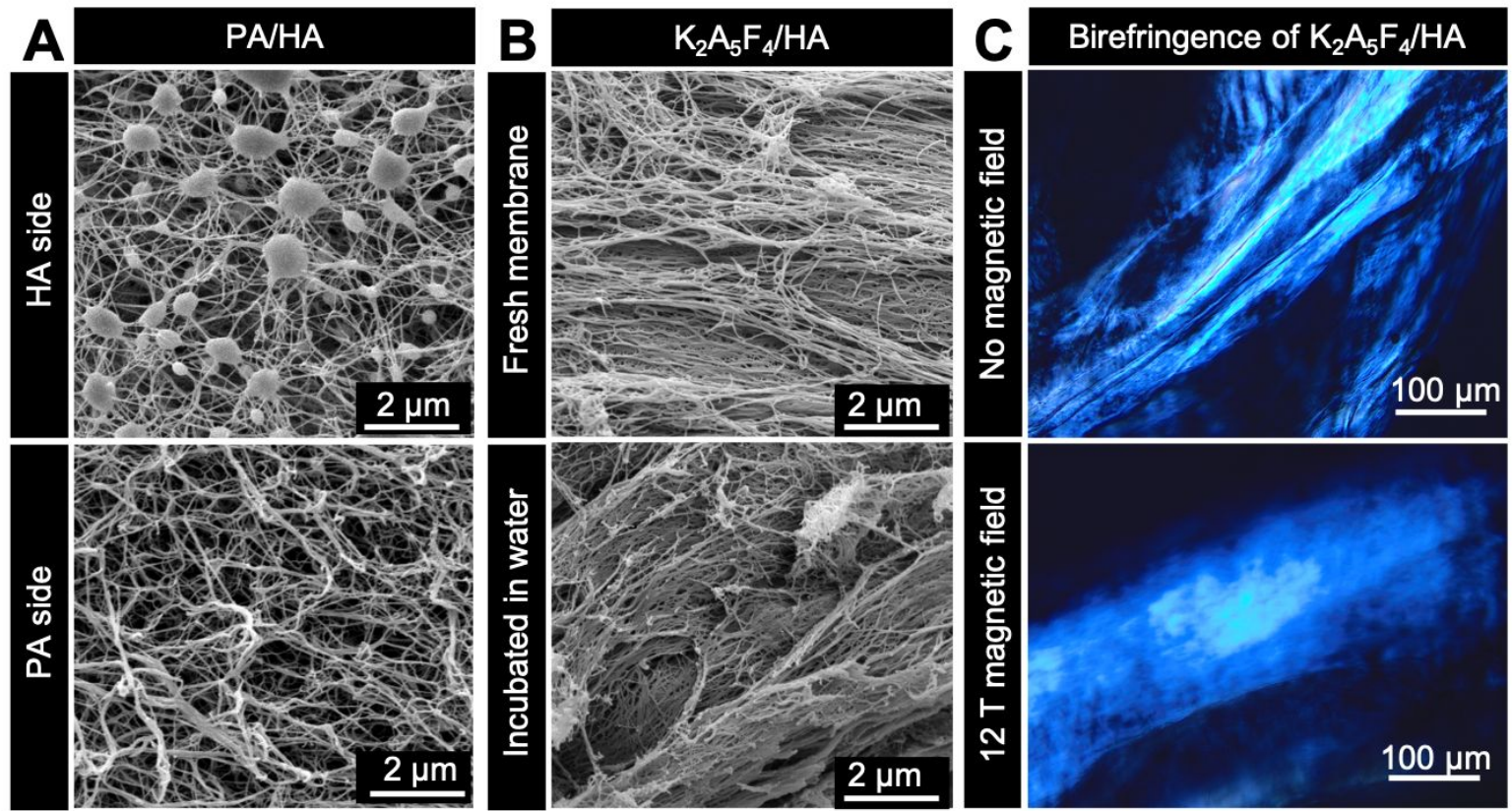

Figure S11. SEM images of peptide/HA membranes assembled under $12 \mathrm{~T}$ magnetic field for 24 hours using a positively charged peptide amphiphile (A) showing random fibres, or with $\mathrm{K}_{2} \mathrm{~A}_{5} \mathrm{~F}_{4}$ peptide (B) displaying aligned nanofibers which are retained after incubation in water for more than a month; (C) Polarized light microscopy images of membranes assembled under and away the magnetic field showing strong birefringence when the nanofibers are aligned on the membrane.

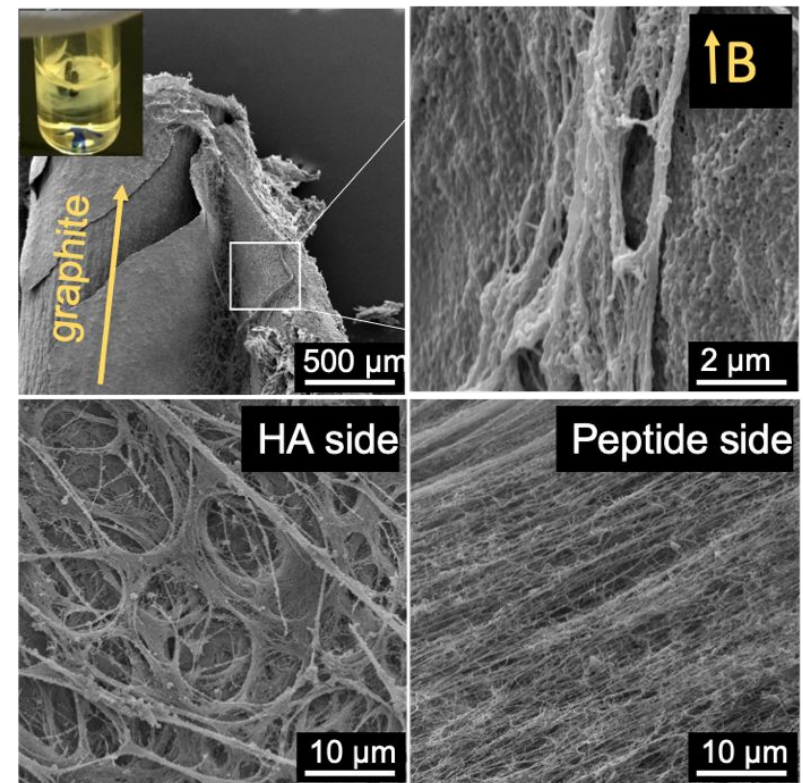

Figure S12. $\mathrm{K}_{2} \mathrm{~A}_{5} \mathrm{~F}_{4} / \mathrm{HA}$ membrane assembled with a graphite rod under $12 \mathrm{~T}$ magnetic field for 24 hours. SEM images showing random fibres on $\mathrm{HA}$ side and aligned fibres on $\mathrm{K}_{2} \mathrm{~A}_{5} \mathrm{~F}_{4}$ peptide side.

References

[1] I. Breßler, J. Kohlbrecher, A. F. Thünemann, J. Appl. Crystallogr. 2015, 48, 1587.

[2] C. J. Bowerman, W. Liyanage, A. J. Federation, B. L. Nilsson, Biomacromolecules 2011, 12, 2735. 
